\title{
Characterization of 2-Path Product Signed Graphs with Its Properties
}

\author{
Deepa Sinha and Deepakshi Sharma \\ Department of Mathematics, South Asian University, Akbar Bhawan Chanakyapuri, New Delhi 110021, India \\ Correspondence should be addressed to Deepa Sinha; deepasinha2001@gmail.com
}

Received 9 March 2017; Accepted 22 May 2017; Published 6 July 2017

Academic Editor: Silvia Conforto

Copyright (C) 2017 Deepa Sinha and Deepakshi Sharma. This is an open access article distributed under the Creative Commons Attribution License, which permits unrestricted use, distribution, and reproduction in any medium, provided the original work is properly cited.

\begin{abstract}
A signed graph is a simple graph where each edge receives a sign positive or negative. Such graphs are mainly used in social sciences where individuals represent vertices friendly relation between them as a positive edge and enmity as a negative edge. In signed graphs, we define these relationships (edges) as of friendship (“+” edge) or hostility (“-” edge). A 2-path product signed graph S $\widehat{\# S}$ of a signed graph $S$ is defined as follows: the vertex set is the same as $S$ and two vertices are adjacent if and only if there exists a path of length two between them in $S$. The sign of an edge is the product of marks of vertices in $S$ where the mark of vertex $u$ in $S$ is the product of signs of all edges incident to the vertex. In this paper, we give a characterization of 2-path product signed graphs. Also, some other properties such as sign-compatibility and canonically-sign-compatibility of 2-path product signed graphs are discussed along with isomorphism and switching equivalence of this signed graph with 2-path signed graph.
\end{abstract}

\section{Introduction}

Signed graph forms one of the most vibrant areas of research in graph theory and network analysis due to its link with behavioural and social sciences. The earliest appearance of signed graphs can be traced back to Heider [1] and Cartwright [2]. From that time to recently, signed theory has evolved rapidly with signed graphs being linked to algebra [3-5], social networks $[6,7]$, other models $[8,9]$, and graph spectra [10] to name few. In graph theory, itself signed graphs have been used to define many properties and new concepts. In $[11,12]$ the signed graph of line signed graphs is discussed, whereas $[13,14]$ talks about common edge signed graphs. The work in $[15,16]$ generalises the $(k, d)$-graceful graphs to signed graphs. The colouring of signed graphs is reported in [17-19]. The connection between the intersection graphs of neighborhood and signed graphs has also been studied [2024]. Recently a Coxeter spectral analysis and a Coxeter spectral classification of the class of edge-bipartite graphs (that is a class of signed (multi)graphs) is developed in the papers [2527] in relation to Lie theory problems, quasi Cartan matrices, Dynkin diagrams, Hilbert's X Problem, combinatorics of
Coxeter groups, and the Auslander-Reiten theory of module categories and their derived categories. In this paper, we were mainly driven to carry out work in the area of signed graphs derived from 2-path product operations, which primarily deals with the structural reconfiguration of the structure of dynamical systems under prescribed rules and the rules are designed to address a variety of interconnections among the elements of the system. We have obtained some theoretical results (some of which are presented in [28]) with a hope of building necessary conceptual resources for applications. For standard terminology and notation in graph theory one can refer to Harary [29] and West [30] and for signed graph literature one can read Zaslavsky [19, 31, 32]. Throughout the text, we consider finite, undirected graph with no loops or multiple edges.

A signed graph is an ordered pair $S=(\Sigma, \sigma)$, where $\Sigma$ is a graph $\Sigma=(V, E)$, called the underlying graph of $S$ and $\sigma: E \rightarrow\{+,-\}$ is a function from the edge set $E$ of $\Sigma$ into the set $\{+,-\}$, called the signature (or sign in short) of $S$. Alternatively, the signed graph can be written as $S=(V, E, \sigma)$, with $V, E$, and $\sigma$ in the above sense. A signed graph is all-positive (resp., all negative) if all its edges are positive (negative); 
further, it is said to be homogeneous if it is either all-positive or all negative and heterogeneous otherwise. The positive (negative) degree of a vertex $v \in S$ denoted by $d^{+}(v)\left(d^{-}(v)\right)$ is the number of positive (negative) edges incident on the vertex $v$ and $d(v)=d^{+}(v)+d^{-}(v)$. The negation of a signed graph $\eta(S)$ is obtained by reversing the sign of edges of $S$. Let $v$ be an arbitrary vertex of a graph $S$. We denote the set consisting of all the vertices of $\sum$ adjacent to $v$ by $N(v)$. This set is called the neighborhood set of $v$ and sometimes we call it as neighborhood of $v$. A marked signed graph is an ordered pair $S^{\mu}=(S, \mu)$ where $S=(\Sigma, \sigma)$ is a signed graph and $\mu: V(\Sigma) \rightarrow\{+,-\}$ is a function from the vertex set $V(\Sigma)$ of $\Sigma$ into the set $\{+,-\}$, called a marking of $S$. $\mathscr{M}_{S}$ denotes the set of all markings on vertices of $S$. For any vertex $v \in S, \mu_{1}(v)=\prod_{u \in N(v)} \sigma(u v)$ is called canonical marking. The marking on the vertices will be specified in the whole text as the case may be.

$N_{*}(t)=\left\{v_{1}^{\mu} \in\left(V\left(S^{\mu}\right)\right): t v\right.$ is an edge with sign $\left.\mu\right\}$, $N_{*}^{+}(t)=\left\{v^{+} \in\left(V\left(S^{\mu}\right)\right): t v\right.$ is an edge $\}$, and $N_{*}^{-}(t)=\left\{v^{-} \epsilon\right.$ $\left(V\left(S^{\mu}\right)\right): t v$ is an edge $\}$. A vertex with a marking $\mu$ is denoted by $v^{\mu}$. A cycle in a signed graph $S$ is said to be positive if the product of the signs of its edges is positive or, equivalently, if the number of negative edges in it is even. A cycle which is not positive is said to be negative.

A signed graph is line balanced or balanced if all its cycles are positive. The partition criterion to characterize the balance property of a signed graph is given by Harary. A marked graph is vertex or point balanced if it does not contain odd number of negative vertices. A signed graph $S$ is signcompatible [35] if there exists a marking $\mu$ of its vertices such that the end vertices of every negative edge receive "-" marks in $\mu$ and no positive edge in $S$ has both of its ends assigned "_" mark in $\mu$; it is sign-incompatible otherwise. A canonically marked graph $S$ is said to be canonically sign-compatible (or $C$ sign-compatible) if end vertices of every negative edge receive "-" sign and no positive edge has both of its ends assigned “-” under $\mu$.

The idea of switching a signed graph was introduced by Abelson and Rosenberg [36] in connection with structural analysis of social behaviour and may be formally stated as follows: given a marking $\mu$ of a signed graph $S$, switching $S$ with respect to $\mu$ is the operation of changing the sign of every edge of $S$ to its opposite whenever its end vertices are of opposite signs in $S_{\mu}$ (also see Gill and Patwardhan $[37,38]$ ). The signed graph obtained in this way is denoted by $(S)_{\mu}$ and is called the $\mu$-switched signed graph or just switched signed graph when the marking is clear from the context. Further, a signed graph $S_{1}$ switches to signed graph $S_{2}$ (or that they are switching equivalent to each other), written as $S_{1} \sim S_{2}$, whenever there

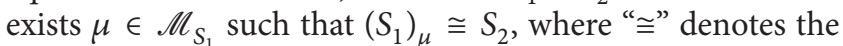
isomorphism between any two signed graphs in the standard sense. Two signed graphs $S_{1}$ and $S_{2}$ are cycle isomorphic if there exists an isomorphism $f: \Sigma_{1} \rightarrow \Sigma_{2}$, where $\Sigma_{1}$ and $\Sigma_{2}$ are underlying graph of $S_{1}$ and $S_{2}$, respectively, such that the sign of every cycle $Z$ in $S_{1}$ equals the sign of $f(Z)$ in $S_{2}$.

Assume that $S=(V, E, \sigma)$ is a signed graph. We associate with $S$ the 2-path signed graph [39] $S \# S=\left(V, E^{\prime}, \sigma^{\prime}\right)$ defined as follows: the vertex set is same as the original signed graph $S$ and two vertices $u, v \in V(S \# S)$, are adjacent if and only if

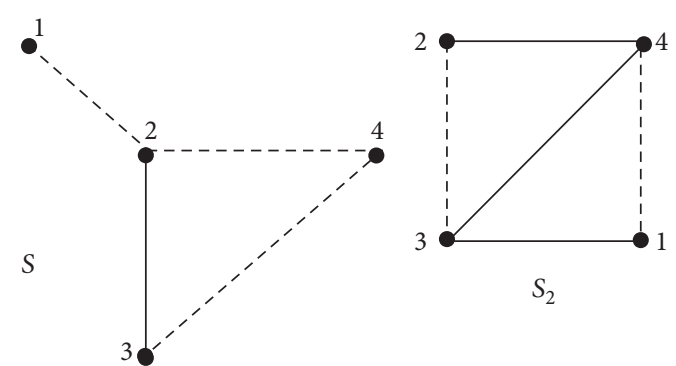

FIGURE 1: A signed graph and its 2-path signed graph.

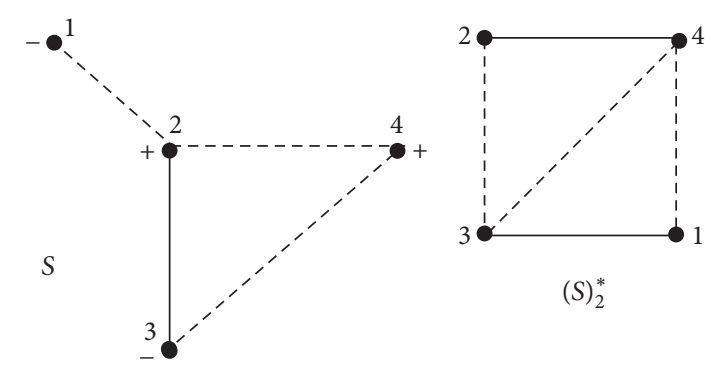

FIGURE 2: A signed graph and its 2-path product signed graph.

there exists a path of length two in $S$. The edge $u v \in V(S \# S)$ is negative if and only if all the edges in all the two paths in $S$ are negative otherwise the edge is positive (see Figure 1). The 2-

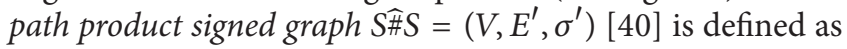
follows: The vertex set is same as the original signed graph $S$ and two vertices $u, v \in V(S \widehat{\# S})$, are adjacent if and only if there exists a path of length two in $S$. The sign $\sigma^{\prime}(u v)=$ $\mu_{1}(u) \mu_{1}(v), \mu_{1}$ is canonical marking (see Figure 2).

Property 1 (see [39]). A 2-subset $\left\{v_{i}, v_{j}\right\}$ in a neighborhood of a vertex in a given signed graph $S$ has property $\mathbf{P}$ if $\left\{v_{i}{ }^{-}, v_{j}{ }^{-}\right\} \subset$ $N_{*}\left(v_{k}\right)$ for some $i, j, k$ and for each $N(t)$ containing $v_{i}, v_{j}$, $\left\{v_{i}^{-}, v_{j}^{-}\right\} \subset N_{*}(t)$.

In the first section, we give a characterization of 2-path product signed graph, followed by a theorem of finding the degree of each vertex in $\widehat{\triangle \# S}$. Also, we find when a 2path product graph is isomorphic and switching equivalent to its negation. Next, we find when $S \widehat{\# S}$ is all negative for a given $S$. The following two sections are dedicated to signed graph properties sign-compatibility and canonicalsign-compatibility. The last section deals with the isomorphism and switching equivalence of the two types of 2-path graphs of signed graphs.

\section{Characterization of 2-Path Product Signed Graph}

We require the following theorems for the characterization of 2-path product signed graph.

Theorem 2 (see [41]). A signed graph $S$ is vertex balanced if and only if it is possible to assign signs to the edges of S such that 
the mark of any vertex $u$ is equal to the product of the signs of the edges incident to $u$.

The following characterization of 2-path graphs was given by Acharya and Vartak.

Theorem 3 (see [42]). A connected graph $\Sigma$ with vertices $v_{i}, i=1, \ldots, n$ is of the 2-path graph form $\Sigma=H \# H$, with some graph $H$ if and only if $\Sigma$ contains a collection of complete subgraphs $\Sigma_{1}, \Sigma_{2}, \ldots, \Sigma_{n}$ such that for each $i, j=1, \ldots, n$

(i) $v_{i} \notin \Sigma_{i}$;

(ii) $v_{i} \in \Sigma_{j} \Leftrightarrow v_{j} \in \Sigma_{i}$;

(iii) $v_{i} v_{j} \in \Sigma$ and there exists $\Sigma_{k}$ containing $v_{i} v_{j}$.

Theorem 4 (see [39]). A connected sigraph $S$ with vertices $v_{i}, i=1, \ldots, n$ is a 2-path sigraph of some sigraph $S^{\prime}$ if and only if $S$ contains a collection of complete subsigraphs $S_{1}, S_{2}, \ldots, S_{n}$ with marked vertices $v_{i}{ }^{\mu}, \mu \in\{+,-\}$ such that, for each $i, j=$ $1, \ldots, n$, the following hold:

(i) $v_{i}^{\mu} \notin S_{i}$;

(ii) $v_{i}{ }^{\mu_{1}} \in S_{j} \Leftrightarrow v_{j}{ }^{\mu_{2}} \in S_{i}, i \neq j, \mu_{1}=\mu_{2}$;

(iii) $v_{i} v_{j} \in E(S)$ with sign $\sigma$; then there exists $S_{k}$ containing $v_{i}^{\mu_{i}}, v_{j}^{\mu_{j}}$ where $\mu_{i}, \mu_{j} \in\{+,-\}$ and if $\sigma\left(v_{i}, v_{j}\right)=-$ then $\left\{v_{i}, v_{j}\right\}$ is a $\mathbf{P}$ pair in $S_{k}$.

The following proposition is evident from $[43,44]$.

Proposition 5. 2-path product signed graph of a signed graph $S$ is always balanced. graph.

We give a characterization for 2-path product signed

Theorem 6. A connected signed graph $S$ with vertices $v_{i}, i=$ $1, \ldots, n$ is of the 2-path product signed graph form $S=S^{\prime} \mathbb{\#} S^{\prime}$ with some signed graph $S^{\prime}$ if and only if the underlying graph $\Sigma$ is a 2-path graph and $S$ is both line balanced and vertex balanced.

Proof.

Necessity. Suppose $S$ is of the 2-path product signed graph form $S=S^{\prime} \cong S^{\prime}$ with vertices $v_{1}, v_{2}, \ldots, v_{n}$. Now from Theorem 3 , there exist $n$ complete subsigned graphs such that (i), (ii), and (iii) hold. Let us consider the set $N(v)$ of neighborhood of a vertex $v$ in $S^{\prime}$. For each vertex $v$ in $S^{\prime}$ there is a neighborhood $N(v)$, hence $n$ such subsets of neighborhoods. Clearly since we consider open neighborhood, $v \notin N(v)$, also if a vertex $u \in N(v)$, then $u v$ is an edge in $S$ and hence $v \in N(u)$. And if $u v$ is an edge in $S$ then $u$ and $v$ are adjacent to a vertex $w$ in $S^{\prime}$. That is $u, v \in N(w)$ such that $\sigma(u v)=\mu_{1}(u) \mu_{1}(v)$ since each vertex has a marking in $S^{\prime}$. We know that $S^{\prime}$ is a canonically marked signed graph; thus each vertex has a marking $\mu_{1}$. Now let $N_{*}\left(v_{i}\right)$ be the neighborhood of a vertex $v_{i}$ with marked vertices retaining the marking from $S^{\prime}$. Then clearly since all three properties (i), (ii), and (iii) of Theorem 3 are satisfied and also by Theorem 2, and Proposition 5, $S$ is line balanced and vertex balanced.

Sufficiency. Let $S$ be a given signed graph such that its underlying graph $\Sigma$ is a 2-path graph and $S$ is both line balanced and vertex balanced. Then by Theorem 3 , it can be written as the union of $n$ complete subsigned graphs $S_{1}, S_{2}, \ldots, S_{n}$ of marked vertices such that for each $i, j=$ $1, \ldots, n$, (i), (ii), and (iii) hold. Now associate a vertex $v_{i} \notin S_{i}$ to $S_{i}$ and join $v_{i}$ to all the vertices in $S_{i}, i=1, \ldots, n$ and giving the edge $v_{i} v_{j}$ sign as that of the product of marking on $v_{i}$ and $v_{j}$ where $v_{j} \in S_{i}$. Let the signed graph thus obtained be $S^{\prime}$. Next we show that $S^{\prime} \widehat{\#} S^{\prime} \cong S$. Obviously $\Sigma^{\prime} \widehat{\#} \Sigma^{\prime} \cong \Sigma$, where $\Sigma^{\prime}$ and $\Sigma$ are underlying graph of $S^{\prime}$ and $S$, respectively. Let $v_{i} v_{j}$ be an edge $S$ with the sign $\sigma$; then $\sigma=\mu_{1}\left(v_{i}\right) \mu_{1}\left(v_{j}\right)$, where $\mu_{1}\left(v_{i}\right)$ and $\mu_{1}\left(v_{j}\right)$ are markings on $v_{i}$ and $v_{j}$, respectively. By hypothesis, $v_{i} v_{j} \in S_{k}$ for some $k$. Hence we will associate a vertex $v_{k}$ to $S_{k}$ and let its marking be $\mu_{1}$. By definition, the sign of edge $v_{i} v_{j}$ in $S^{\prime} \widehat{\#} S^{\prime}$ is $\sigma^{\prime}, \sigma^{\prime}=\mu_{1}\left(v_{i}\right) \mu_{1} \mu_{1}\left(v_{j}\right) \mu_{1}$. That is $\sigma^{\prime}=\sigma=\mu_{1}\left(v_{i}\right) \mu_{1}\left(v_{j}\right)$. Therefore, $S^{\prime}$ is the signed graph such that $S^{\prime} \mathbb{\#} S^{\prime} \cong S$.

The characterization of 2-path signed graph in Theorem 4 provides us with a mechanism to check if a given signed graph is 2-path of some signed graph, which is discussed in Algorithm 1. This has been rigorously studied elsewhere in the author's contribution which is fully devoted to 2-path signed graphs and its properties. Thus Algorithm 2 using Algorithm 1 detects if the given signed graph is 2-path product signed graph and find the original signed graph. In Algorithm 2, we use the adjacency matrix $A=\{a[i][j]: i, j \leq n\}$ and its order $n$ to find the original signed graph. Algorithm 3 is used to find the 2-path product signed graph for a given signed graph.

Theorem 7. If $u^{\mu_{1}} \in V\left(S_{\mu_{1}}\right), \mu_{1} \in\{+,-\}$ being the canonical

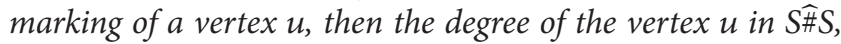
for a given signed graph $S$, is given by the following:

(i) If $\mu_{1}=+$ then positive degree of $u$ in $\widehat{S \# S=}$ $\left|\bigcup_{u^{+} \in N_{*}(x)}\left(N_{*}^{+}(x)-\{u\}\right)\right|$ and the negative degree of $u=\left|\bigcup_{u^{+} \in N_{*}(x)}\left(N_{*}^{-}(x)-\{u\}\right)\right|$.

(ii) If $\mu_{1}=-$ then positive degree of $u$ in $\widehat{S \# S=}$ $\left|\bigcup_{u^{-} \in N_{*}(x)}\left(N_{*}^{-}(x)-\{u\}\right)\right|$ and the negative degree is $\left|\bigcup_{u^{-} \in N_{*}(x)}\left(N_{*}^{+}(x)-\{u\}\right)\right|$.

Proof. By Theorem 6 the neighborhoods of a vertex of $S$ gives

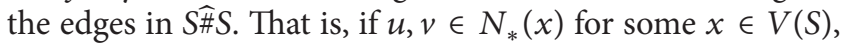

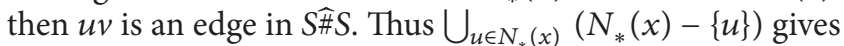
the number of vertices which form an edge with $u$ in $S \widehat{\# S}$. And since the marking is canonical in $S$ thus positive edges in $\widehat{S \# S}$ are given by vertices with same marking. Thus a vertex $u^{\mu_{1}}, \mu_{1} \in\{+,-\}$ in $V(S \widehat{\# S})$ is given by the following:

(i) If $\mu_{1}=+$ then positive degree of $u$ in $\widehat{\# \# S=}$ $\left|\bigcup_{u^{+} \in N(x)}\left(N_{*}^{+}(x)-\{u\}\right)\right|$ and the negative degree of $u=\left|\bigcup_{u^{+} \in N_{*}(x)}\left(N_{*}^{-}(x)-\{u\}\right)\right|$. 
Input. The adjacency matrix $A_{n \times n}=\left\{a_{i j} \in\{-1,0,+1\}: 1 \leq i, j \leq n\right\}$

Output. If $A$ is a 2-path for some signed graph $A^{\prime}$ then returns $A^{\prime}$.

Process

(1) Collect all the cliques $D$ for each vertex $n$, using Bron-Kerbosch algorithm [33].

(2) Mark every vertex by + and then - in each clique.

(3) Calculate $B$, which represent the all possible combinations generated by each marked vertex from the clique.

(4) for $t=1$ to $\operatorname{size}(B)$ do

(5) $\quad$ Select $B_{t} \in B$

(6) for $k=1$ to $n$ do

(7) Select $m \in B_{t} \& \& n^{\prime} \in B_{t}$

(8) if $a[m]\left[n^{\prime}\right] \neq 0$ then

(9) $\quad E(k)=B_{t}$

(10) for $k=1$ to $n$ do

(11) $\quad$ for $l=1$ to $n$ do

(12)

(13)

(14)

(15)

(16)

(17)

(18)

(26) For all the combinations of elementary swamping operations on either rows or columns in $A$, repeat (4).

(27) If all the combinations are checked and no such matrix $C$ is obtained then no such graph exist.

(28) If such a signed graph exist then $C$ is the adjacency matrix of required signed graph whose 2-path signed graph is $S$.

Algorithm 1: To check if the given signed graph is a 2-path of some other signed graph.

Input. The adjacency matrix $A$ of signed graph $S$ and dimension $n$

Output. If $S$ is a 2-path for some signed graph then returns its adjacency matrix $A^{\prime}$.

Process

(1) We use Algorithm 1 to detect if $A$ is a 2-path signed graph.

(2) Use algorithm in [34] to check if $S$ is balanced.

(3) for $i=1$ to $n$ do

(4) $d[i]=1$; for $j=1$ to $n$ do

(5) if $a[i][j] \neq 0$ then

(6) $\quad d[i]=d[i] * a[i][j]$;

(7) $f=1$;

(8) for $i=1$ to $n$ do

(9) $f=f * d[i]$

(10) if $f=-1$ then

(11) The given signed graph is not a 2-path product signed graph

(12) else

(13) The given signed graph is a 2-path product signed graph

Algorithm 2: To check if the given signed graph is a 2-path product of some other signed graph. 
Input. Adjacency matrix $A$ and dimension $n$.

Output. Adjacency matrix of 2-path signed graph

Process

(1) Enter the order $n$ and adjacency matrix $A$ of for a given signed graph $S$.

(2) Collect all the $q \mathbf{P}$ pairs for given signed graph $S$.

(3) for $i=1$ to $n$ do

(4) $d[i]$ for $j=1$ to $n$ do

(5) $\quad b[i][j]=0$ if $a[i][j] \neq 0$ then

(6) $d[i]=d[i] * a[i][j]$;

(7) for $i=1$ to $n$ do

$d[i]=d[i] * a[i][j]$

(8) for $j=1$ to $n$ do

(9) for $k=1$ to $n$ do

(10) $\quad$ if $(a[i][j] \neq 0) \& \&(a[i][k] \neq 0)$ then

(11) $\quad$ if $(d[k] * d[j]=-1)$ then

(12) $\quad b[k][j]=-1$

(13) $\quad b[j][k]=-1$

(14)

(15)

(16)

else

$$
b[k][j]=1
$$

$b[j][k]=1$

Algorithm 3: Algorithm to obtain a 2-path product signed graph for a given signed graph.

(ii) If $\mu_{1}=-$ then positive degree of $u$ in $S \widehat{\#} S=$ $\left|\bigcup_{u^{-} \in N_{*}(x)}\left(N_{*}^{-}(x)-\{u\}\right)\right|$ and the negative degree is $\left|\bigcup_{u^{-} \in N_{*}(x)}\left(N_{*}^{+}(x)-\{u\}\right)\right|$.

Theorem 8. $S \mathbb{\#} S \cong \eta(S) \widehat{\#} \eta(S)$, if and only if $S$ is a signed graph with each vertex of even degree.

Proof.

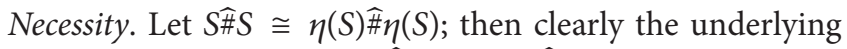

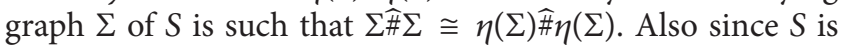
a canonically marked signed graph with each vertex of even degree, the mark on every vertex will be the product of edges incident to it. Let if possible $v$ be a vertex with $x$ number of positive edges incident to $v$ and $y$ be the number of negative edges incident to it. Then one of the following cases arises.

Case 1. Let $x$ be even; then $y$ is also even since the total number of edges incident to $v$ is even. In negation of $S, y$ will again be even (since $x$ is even in $S$ ). Thus both retain the same marking for $v$.

Case 2. Let $x$ be odd then $y$ is odd. Clearly $\mu_{1}(v)=-$; also $\mu_{1}(v)$ in $\eta(S)$ is again negative. Thus in both $S \# S$ and $\eta(S) \widehat{\#} \eta(S)$ the marking of $v$ is - .

Clearly, since marking on each vertex remains the same so their 2-path product signed graphs remain isomorphic.

Sufficiency. Let $S \widehat{\#} S \cong \eta(S) \widehat{\#} \eta(S)$. Let if possible $v$ be a vertex with odd degree. Let $x$ be the number of positive edges incident to $v$ and $y$ be the negative edges incident to $v$; then the following cases arise:

(i) If $x$ is odd then $y$ is even. Consequently, $v$ receives a positive marking in $S$, but in its negation the number of negative edges becomes odd and hence the sign is reversed.

(ii) If $x$ is even then $y$ is odd. The marking in $S$ and $\eta(S)$ is again reversed.

Thus if the signed graph has odd degree vertices then the 2path product graphs of $S$ and $\eta(S)$ are not isomorphic, which is a contradiction.

Corollary 9. For any signed graph $S, S \widehat{\#} S \sim \eta(S) \widehat{\#} \eta(S)$.

Proof. Clearly, $\Sigma \widehat{\#} \Sigma \cong \eta(\Sigma) \widehat{\#} \eta(\Sigma)$, where $\Sigma$ is underlying graph of $S$. Next we know that $S \widehat{\#} S$ is always balanced, for every signed graph $S$. Thus all cycles are positive and have even number of negative edges. Thus both $S \mathbb{\#} S$ and $\eta(S) \widehat{\#} \eta(S)$ will possess cycles with even number of negative edges. Thus $S \widehat{\# S} \sim \eta(S) \widehat{\#} \eta(S)$.

Theorem 10. A 2-path product signed graph S $\cong S$ of a given signed graph $S$ is all negative if and only if $S$ is either a cycle of length $4 m$ or a signed path and $S$ does not contain a subsigned path $u^{+}, w^{\mu_{1}}, v^{+}$or $u^{-}, w^{\mu_{1}}, v^{-}$, in $S$ where $\mu_{1} \in\{+,-\}$.

Proof.

Necessity. Let for a given $S$ its 2-path product signed graph $S \widehat{\# S}$ be all negative. Clearly, the signed graph $S$ can be a tree or a cycle. Now if $S$ is not a cycle or tree then $S \ddot{\# S}$ will consist of cliques which can not be all negative since cliques always consist of a cycle of length three which can never be all negative as 2-path product signed graphs are always balanced. Clearly, 2-path graph of a cycle of odd length is selfisomorphic. Thus the cycle of odd length can not generate all negative 2-path product graphs. The 2-path graphs of cycles of even length say $2 m$ are disjoint cycles of length $m$ each. So if $m$ is odd then also the 2-path product signed graph can never be all negative. Thus, a cycle of length $4 m$ can generate 


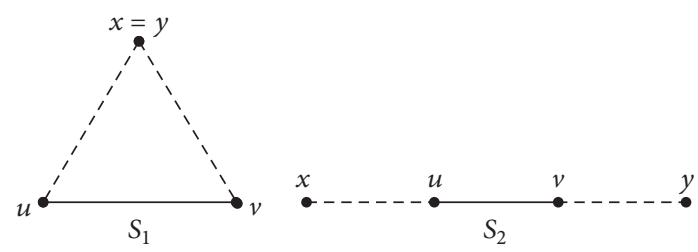

FIGURE 3: Acharya and Sinha two forbidden subsigned graphs for a sign-compatible signed graph.

all negative 2-path product signed graphs. To produce all

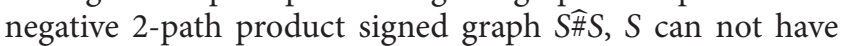
subsigned path $u^{+}, w^{\mu_{1}}, v^{+}$or $u^{-}, w^{\mu_{1}}, v^{-}$, on any subsigned

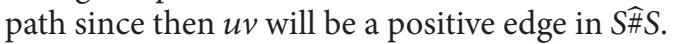

Also if there is a tree $S$ with a vertex of degree greater than two, then clearly it gives rise to a clique containing cycles of length three in $\widehat{\triangle \# S}$, thus having at least one positive edge. Hence the tree can not have a vertex of degree greater than two. Thus, it is a signed path.

Sufficiency. let $S$ is either a cycle of length $4 m$ or a signed path and $S$ does not contain a subsigned path $u^{+}, w^{\mu_{1}}, v^{+}$or $u^{-}, w^{\mu_{1}}, v^{-}$, where $\mu_{1} \in\{+,-\}$. Clearly $\widehat{\triangle \# S}$ will be disjoint cycles in case of cycle except for $m=1$ where it will be two disjoint signed paths. And in case of signed path $\widehat{S \# S \text { will }}$ be disjoint paths. And since always for any subsigned path $u, w, v$ in $S, u$, and $v$ will occupy opposite mark in $S \widehat{\# S}$, thus

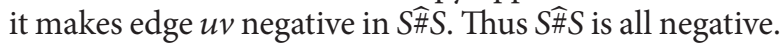

\section{Sign-Compatibility of 2-Path Product Signed Graphs}

In this section, we give a characterization of sign-compatibility for 2-path product signed graphs.

Theorem 11 (see [35]). A signed graph $S$ is sign-compatible if and only if $S$ does not contain a subsigned graph isomorphic to either of the two signed graphs in Figure $3, S_{1}$ formed by taking the path $P_{4}: x, u, v, y$ with both the edges $x u$ and vy negative and the edge uv positive, and $S_{2}$ formed by taking $S_{1}$ and identifying the vertices $x$ and $y$.

Theorem 12. A 2-path product signed graph S\#S of a signed graph $S$ is sign-compatible if and only if

(i) S does not contain a heterogeneous canonically marked triangle or $K_{1,3}$;

(ii) $S$ does not consist of the canonically marked subsigned path $P_{7}: u^{+}, w^{\mu_{1}}, v^{-}, x^{\mu_{1}}, y^{-}, z^{\mu_{1}}, t^{+}$or $\eta\left(P_{7}\right)$ : $u^{-}, w^{\mu_{1}}, v^{+}, x^{\mu_{1}}, y^{+}, z^{\mu_{1}}, t^{-}$, where $\mu_{1} \in\{+,-\}$.

Proof.

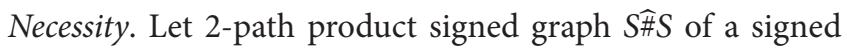
graph $S$ be sign-compatible. To prove (i) and (ii), let $S$ consist of a heterogeneous marked triangle $u, v, w, u$; then there exist two vertices with same mark and one vertex with different mark. Clearly the 2-path product signed graph $\widehat{S \# S}$ will contain triangle $u, v, w, u$ with two negative edges and

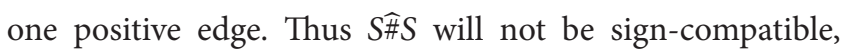
which is a contradiction. Again if $S$ contains a heterogeneous

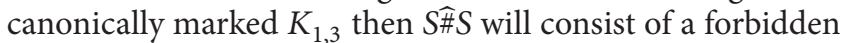
triangle $S_{1}$ in Figure 3. Hence (i) holds. Let if possible $S$ consist of the canonically marked subsigned path $P_{7}: u^{+}$, $w^{\mu_{1}}, v^{-}, x^{\mu_{1}}, y^{-}, z^{\mu_{1}}, t^{+}$or $\eta\left(P_{7}\right): u^{-}, w^{\mu_{1}}, v^{+}, x^{\mu_{1}}, y^{-}, z^{\mu_{1}}, t^{-}$, where $\mu_{1} \in\{+,-\}$. Then $S \mathbb{\#} S$ will contain a forbidden $S_{2}$ in Figure 3; thus $\widehat{\triangle \# S}$ will not be sign-compatible which is a contradiction to our assumption. Hence (ii) holds.

Sufficiency. Let (i) and (ii) hold. To show $\widehat{A} S$ is sign-com-

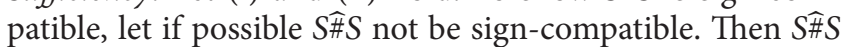
must consist of subsigned graph isomorphic to Figure 3, which is not possible as then either (i) or (ii) does not hold true. Hence $\widehat{S} S$ is sign-compatible.

\section{C-Sign-Compatibility of 2-Path Product Signed Graphs}

This section gives the C-sign-compatibility of 2-path product signed graphs.

Proposition 13 (see [45]). Every C-sign-compatible signed graph is sign-compatible.

Theorem 14 (see [45]). A signed graph $S=(\Sigma, \sigma)$, is $C$-signcompatible if and only if the following holds for $S$ :

(i) For every vertex $v \in V(S)$ either $d^{-}(v)=0$ or $d^{-}(v)=$ $1(\bmod 2)$ and

(ii) For every positive edge $e_{k}=v_{i} v_{j}$ in $S$ either $d^{-}\left(v_{i}\right)=0$ or $d^{-}\left(v_{j}\right)=0$.

Theorem 15. A 2-path product signed graph S $\widehat{\# S}$ of a signed graph $S$ is C-sign-compatible if and only if

(i) $S$ is sign-compatible;

(ii) $S$ does not contain a subsigned path $A=u^{-}, w^{\mu_{1}}, v^{-}$, of vertices $u, w, v$ where $\mu_{1} \in\{+,-\}$;

(iii) if there exist a subsigned path $u^{+}, w^{\mu_{1}}, v^{+}$of vertices $u, w, v$ in $S$; then either $d^{-}(u)=0$ or $d^{-}(v)=0$, where $\mu_{1} \in\{+,-\}$;

Proof.

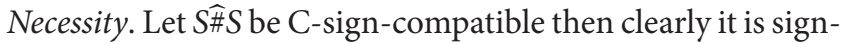
compatible by Proposition 13. Let us suppose $S$ contains a subsigned graph $u^{-}, w^{\mu_{1}}, v^{-}$; then clearly $u v$ is a positive edge in $\widehat{S \# S}$ such that $d^{-}(u) \neq 0$ and $d^{-}(v) \neq 0$, which is a contradiction to the fact that $\widehat{S \# S}$ is $\mathrm{C}$-sign-compatible. Hence $S$ does not contain subsigned path $u^{-}, w^{\mu_{1}}, v^{-}$.

Let there exist a subsigned path $u^{+}, w^{\mu_{1}}, v^{+}$on vertices $u, w, v$ in $S$, such that $d^{-}(u) \neq 0$ and $d^{-}(v) \neq 0$. Then $u v$ is a positive edge in $\widehat{S \# S}$ with both the vertices having negative degrees which is a contradiction to Theorem 14. Thus (i), (ii), and (iii) hold. 
Sufficiency. Let (i), (ii), and (iii) hold. Then clearly for each

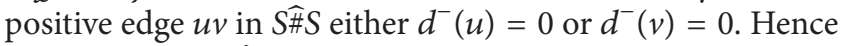
by Theorem 14, $\widehat{S \# S}$ is C-sign-compatible.

\section{Isomorphism and Switching Equivalence of $S \# S$ and $S \# S$}

In this section, we give the switching equivalent and isomorphism for the two definitions of 2-path signed graphs.

Theorem 16 (see [46]). Given a graph G, any two signed graphs are switching equivalent if and only if they are cycle isomorphic.

Theorem 17 (see [39]). For a signed graph $S$ of order $n$, its 2-path signed graph $S \# S$ is balanced if and only if for all sequences of vertices $x_{1}, x_{2}, \ldots, x_{N}, 1 \leq N \leq n$ in $S$ such that $x_{1}, x_{2} \in N\left(t_{1}\right) ; x_{2}, x_{3} \in N\left(t_{2}\right) ; \ldots ; x_{1}, x_{N} \in N\left(t_{N}\right)$ for some $t_{1}, t_{2}, \ldots, t_{N} \in V(S)$; then the pairs $x_{i}, x_{i+1} \in N\left(t_{i}\right), 1 \leq i \leq N$ having property $\mathbf{P}$ are even in each sequence.

Theorem 18. The 2-path signed graph S\#S and 2-path product graph $\widehat{A \# S}$ are switching equivalent if and only if $S \# S$ is balanced.

Proof.

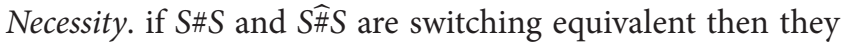
are cycle isomorphic and hence $S \# S$ is balanced.

Sufficiency. Clearly, $\sum \widehat{\#} \Sigma \cong \sum \# \sum$. Next, we know that $S \widehat{\# S}$ is

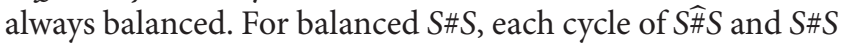
will be positive which implies that $S \widehat{\# S}$ and $S \# S$ will be cycle isomorphic. Thus, by Theorem 16, $\widehat{\#} S$ and $S \# S$ are switching equivalent.

Theorem 19. The 2-path signed graph S\#S and 2-path product graph $\widehat{S \# S}$ are isomorphic, if and only if there exists subsigned path $u^{+}, w^{\mu_{1}}, v^{-}$or $u^{-}, w^{\mu_{1}}, v^{+}, \mu_{1} \in\{+,-\}$ in $S$; then $\{u, v\}$, satisfies $\mathbf{P}$ property.

Proof.

Necessity. For a signed graph $S$, let its 2-path signed graph $S \# S$ and 2-path product graph $S \widehat{\#} S$ be isomorphic; here if $u v$ is a negative (positive) in $S \# S$ then it is negative in $S \widehat{\# S}$. All the pair of vertices $\{u, v\}$ are negative in $S \# S$ and have property $\mathbf{P}$. If there exist subsigned path $u^{+}, w^{\mu_{1}}, v^{-}$and $u^{-}, w^{\mu_{1}}, v^{+}$where $\mu_{1} \in\{+,-\}$ in $S$ then $\mathcal{u v}$ is a negative edge in $\widehat{S \# S}$ and thus $\{u, v\}$ satisfies property $\mathbf{P}$.

Sufficiency. Let there exist subsigned path $u^{+}, w^{\mu_{1}}, v^{-}$and $u^{-}, w^{\mu_{1}}, v^{+}$in $S$ then $\{u, v\}$ has property $\mathbf{P}$. To show 2-

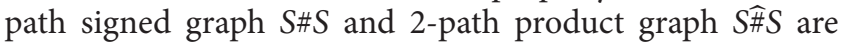
isomorphic. Clearly $\sum \widehat{\#} \Sigma \cong \sum \# \Sigma$, $\Sigma$ being the underlying graph of $S$. Thus we need to show that the sign convention

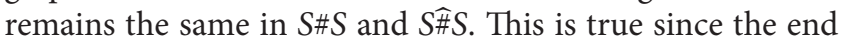
vertices of every negative edge of $\widehat{S} \widehat{\#}$ have property $\mathbf{P}$ and hence $u v$ is a negative edge in $S \# S$. And thus 2-path signed

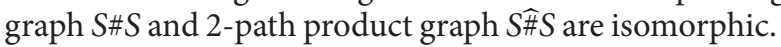

\section{Conclusion}

In this paper, we have worked on 2-path product signed graph of a given signed graph $S$. A 2-path product signed graph is the signed graph where the vertex set is same as the original signed graph $S$ and two vertices $u, v \in V(S \widehat{\# S})$ are adjacent if and only if there exists a path of length two in $S$. The sign $\sigma^{\prime}(u v)=\mu_{1}(u) \mu_{1}(v), \mu_{1}$ being canonical marking. We give its algorithmic characterization along with its properties like sign-compatibility and C-sign-compatibility. Also, we find the isomorphism of 2-path product signed graph and its negation. We next find isomorphism of 2-path signed graph and 2-path product signed graphs.

\section{Conflicts of Interest}

All the authors declare that they have no conflicts of interest regarding publication of this paper.

\section{Acknowledgments}

The authors wish to thank Professor Thomas Zaslavsky, Binghamton University, State University of New York, for going through the paper and giving suggestions. His input to this paper has helped the authors to bring the paper in the present form.

\section{References}

[1] F. Heider, "Attitudes and Cognitive Organization," Journal of Psychology: Interdisciplinary and Applied, vol. 21, no. 1, pp. 107112, 1946.

[2] D. Cartwright and F. Harary, "Structural balance: a generalization of Heider's theory," Psychological Review, vol. 63, no. 5, pp. 277-293, 1956.

[3] T. Zaslavsky, Matrices in the theory of signed simple graphs. https://arxiv.org/abs/1303.3083.

[4] P. Sharma and M. Acharya, "Balanced signed total graphs of commutative rings," Graphs and Combinatorics, vol. 32, no. 4, pp. 1585-1597, 2016.

[5] D. Sinha, D. Ayushi, and B. D. Acharya, "Unitary addition cayley signed graphs," European Journal of Pure and Applied Mathematics, vol. 6, no. 2, pp. 189-210, 2013.

[6] J. Kunegis, Applications of structural balance in signed social networks. https://arxiv.org/abs/1402.6865.

[7] J. Leskovec, D. P. Huttenlocher, and J. M. Kleinberg, "Signed networks in social media," in Proceedings of the 28th International Conference on Human Factors in Computing Systems (CHI '10), pp. 1361-1370, Atlanta, Ga, USA, April 2010.

[8] W. Kager, M. Lis, and R. Meester, "The signed loop approach to the Ising model: foundations and critical point," Journal of Statistical Physics, vol. 152, no. 2, pp. 353-387, 2013.

[9] L. Ou-Yang, D.-Q. Dai, and X.-F. Zhang, "Detecting Protein Complexes from Signed Protein-Protein Interaction Networks," IEEE/ACM Transactions on Computational Biology and Bioinformatics, vol. 12, no. 6, pp. 1333-1344, 2015.

[10] D. Cvetković, P. Rowlinson, and S. Simić, An Introduction to the Theory of Graph Spectra, Cambridge University Press, New York, NY, USA, 2010. 
[11] D. Sinha and P. Garg, "Canonical consistency of semi-total line signed graphs," National Academy Science Letters, vol. 38, no. 5, pp. 429-432, 2015.

[12] K. A. Germina, S. Hameed K., and T. Zaslavsky, "On products and line graphs of signed graphs, their eigenvalues and energy," Linear Algebra and its Applications, vol. 435, no. 10, pp. 24322450, 2011.

[13] M. Acharya and D. Sinha, "Common-edge sigraphs," AKCE International Journal of Graphs and Combinatorics, vol. 3, no. 2, pp. 115-130, 2006.

[14] P. S. Reddy, E. Sampathkumar, and M. S. Subramanya, "Common-edge signed graph of a signed graph," Journal of the Indonesian Mathematical Society, vol. 16, no. 2, pp. 105-113, 2010.

[15] M. Acharya and T. Singh, "Graceful signed graphs," Czechoslovak Mathematical Journal, vol. 54, no. 2, pp. 291-302, 2004.

[16] M. Acharya and T. Singh, "Graceful signed graphs: Ii. the case of signed cycles with connected negative sections," Czechoslovak Mathematical Journal, vol. 55, no. 1, pp. 25-40, 2005.

[17] M. Behzad and G. Chartrand, "Line-coloring of signed graphs," Elemente der Mathematik, vol. 24, pp. 49-52, 1969.

[18] T. Fleiner and G. Wiener, "Coloring signed graphs using dfs," Optimization Letters, vol. 10, no. 4, pp. 865-869, 2016.

[19] T. Zaslavsky, "Signed graphs," Discrete Applied Mathematics, vol. 4, no. 1, pp. 47-74, 1982.

[20] R. Rangarajan, M. S. Subramanya, and P. S. Reddy, "Neighborhood signed graphs," Southeast Asian Bulletin of Mathematics, vol. 36, no. 3, pp. 389-397, 2012.

[21] D. Sinha and A. Dhama, "Negation switching invariant signed graphs," Electronic Journal of Graph Theory and Applications. EJGTA, vol. 2, no. 1, pp. 32-41, 2014.

[22] P. S. K. Reddy, P. N. Samanta, and P. S. Kavita, "On signed graphs whose two path signed graphs are switching equivalent to their jump signed graphs," Mathematical Combinatorics, vol. 1, pp. 74-79, 2015.

[23] D. Sinha and D. Sharma, "Algorithmic characterization of signed graphs whose two path signed graphs and square graphs are isomorphic," in Proceedings of the 2014 International Conference on Soft Computing Techniques for Engineering and Technology, ICSCTET 2014, IEEE, pp. 1-5, August 2014.

[24] D. Sinha and D. Sharma, "On Square and 2-path Signed Graph," Journal of Interconnection Networks, vol. 16, no. 1, Article ID 1550011, 2016.

[25] D. Simson, "Symbolic algorithms computing GRAm congruences in the Coxeter spectral classification of edge-bipartite graphs, I. A GRAm classfication," Fundamenta Informaticae, vol. 145, no. 1, pp. 19-48, 2016.

[26] A. Mroz, "Congruences of edge-bipartite graphs with applications to Grothendieck group recognition I. inflation algorithm revisited," Fundamenta INFormaticae, vol. 146, no. 2, pp. 121144, 2016.

[27] A. Mroz, "Congruences of edge-bipartite graphs with applications to grothendieck group recognition ii. coxeter type study," Fundamenta Informaticae, vol. 146, no. 2, pp. 145-177, 2016.

[28] D. Sinha and S. Deepakshi, "2-path product signed graph," in Proceedings of the National Conference on Algebra, Analysis, Coding and Cryptography, October 2016.

[29] F. Harary, Graph Theory, Addison-Wesley, Reading, Mass, USA, 1969.

[30] D. B. West, Introduction to Graph Theory, Prentice hall Upper, Saddle River, NJ, USA, 2001.
[31] T. Zaslavsky, "Signed analogs of bipartite graphs," Discrete Mathematics, vol. 179, no. 1-3, pp. 205-216, 1998.

[32] T. Zaslavsky, "A mathematical bibliography of signed and gain graphs and allied areas," Electronic Journal of Combinatorics, vol. 1000:DS8, 2012.

[33] C. Bron and J. Kerbosch, "Algorithm 457: finding all cliques of an undirected graph," Communications of the ACM, vol. 16, no. 9, pp. 575-577, 1973.

[34] F. Harary and J. A. Kabell, "A simple algorithm to detect balance in signed graphs," Mathematical Social Sciences, vol. 1, no. 1, pp. 131-136, 1980.

[35] D. Sinha and Ai. Dhama, "Sign-compatibility of some derived signed graphs," Mapana-Journal of Sciences, vol. 11, no. 4, p. 14, 2012.

[36] R. P. Abelson and M. J. Rosenberg, "Symbolic psycho-logic: A model of attitudinal cognition," Behavioral Science, vol. 3, no. 1, pp. 1-13, 1958.

[37] M. K. Gill and G. A. Patwardhan, "Switching invariant two-path signed graphs," Discrete Mathematics, vol. 61, no. 2-3, pp. 189196, 1986.

[38] M. K. Gill and G. A. Patwardhan, "A characterization of sigraphs which are switching equivalent to their line sigraphs," Journal of Mathematical and Physical Sciences, vol. 15, no. 6, pp. 567-571, 1981.

[39] D. Sinha and D. Sharma, "On 2-path signed graphs," in Proceedings of the 2016 International Workshop on Computational Intelligence (IWCI), IEEE, pp. 218-220, Dhaka, Bangladesh, December 2016.

[40] P. S. K. Reddy and M. S. Subramanya, "Note on path signed graphs," Notes on Number Theory and Discrete Mathematics, vol. 4, no. 16, p. 1, 2009.

[41] E. Sampathkumar, "Point signed and line signed graphs," National Academy Science Letters, vol. 7, no. 3, pp. 91-93, 1984.

[42] A. B. Devadas and M. N. Vartak, "Open neighborhood graphs," Tech. Rep. 7, Indian Institute of Tech- nology Department of Mathematics Research Report, 1973.

[43] J. A. Davis, "Clustering and structural balance in graphs," Social networks. A developing paradigm, pp. 27-34, 1977.

[44] F. Harary, "On the notion of balance of a signed graph," The Michigan Mathematical Journal, vol. 2, no. 2, pp. 143-146, 1953.

[45] D. Sinha and A. Dhama, "Canonical-sign-compatibility of some signed graphs," Journal of Combinatorics Information \& System Sciences, vol. 38, no. 1-4, 129 pages, 2013.

[46] T. Sozanśki, "Enumeration of weak isomorphism classes of signed graphs," Journal of Graph Theory, vol. 4, no. 2, pp. 127144, 1980. 

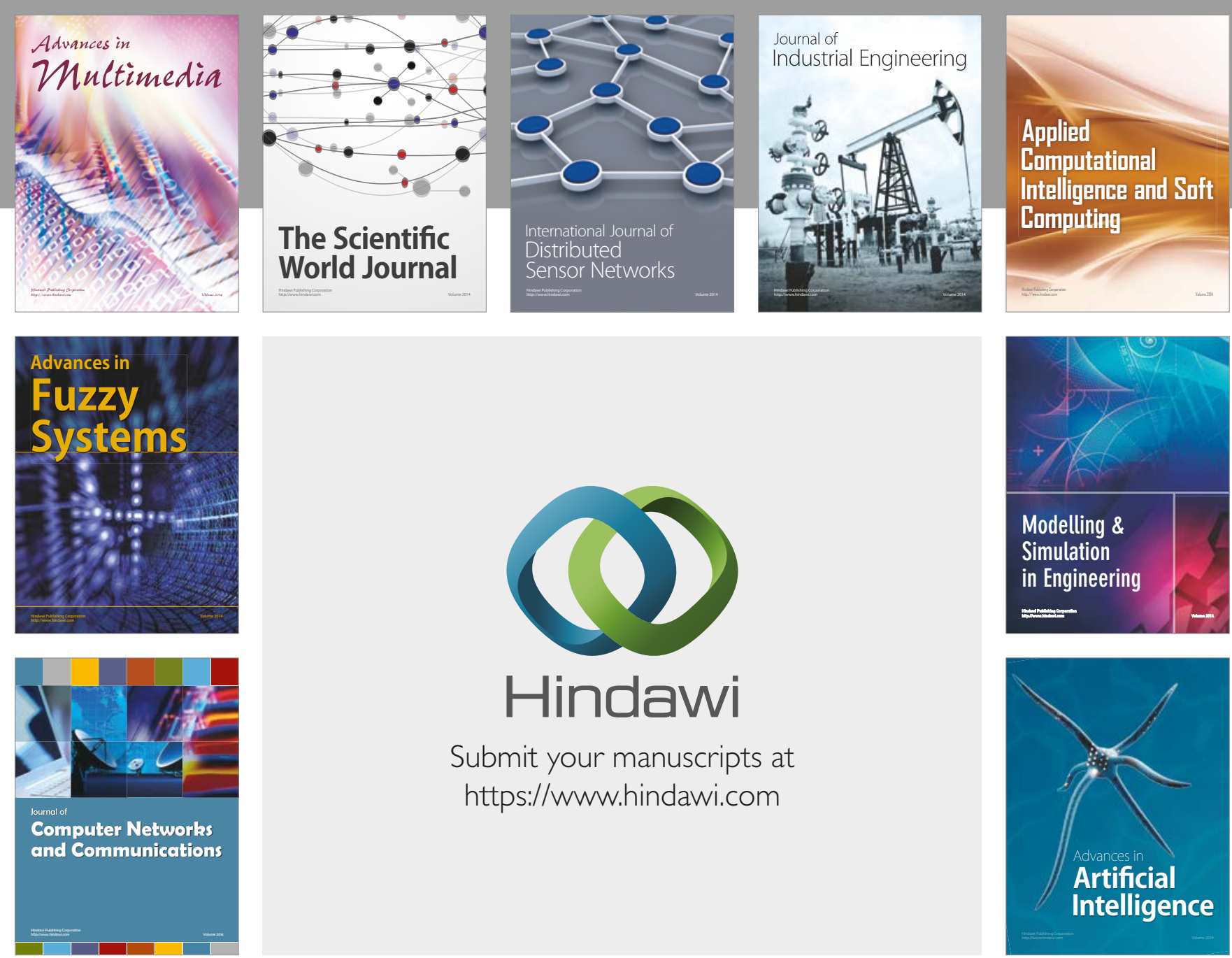

\section{Hindawi}

Submit your manuscripts at

https://www.hindawi.com
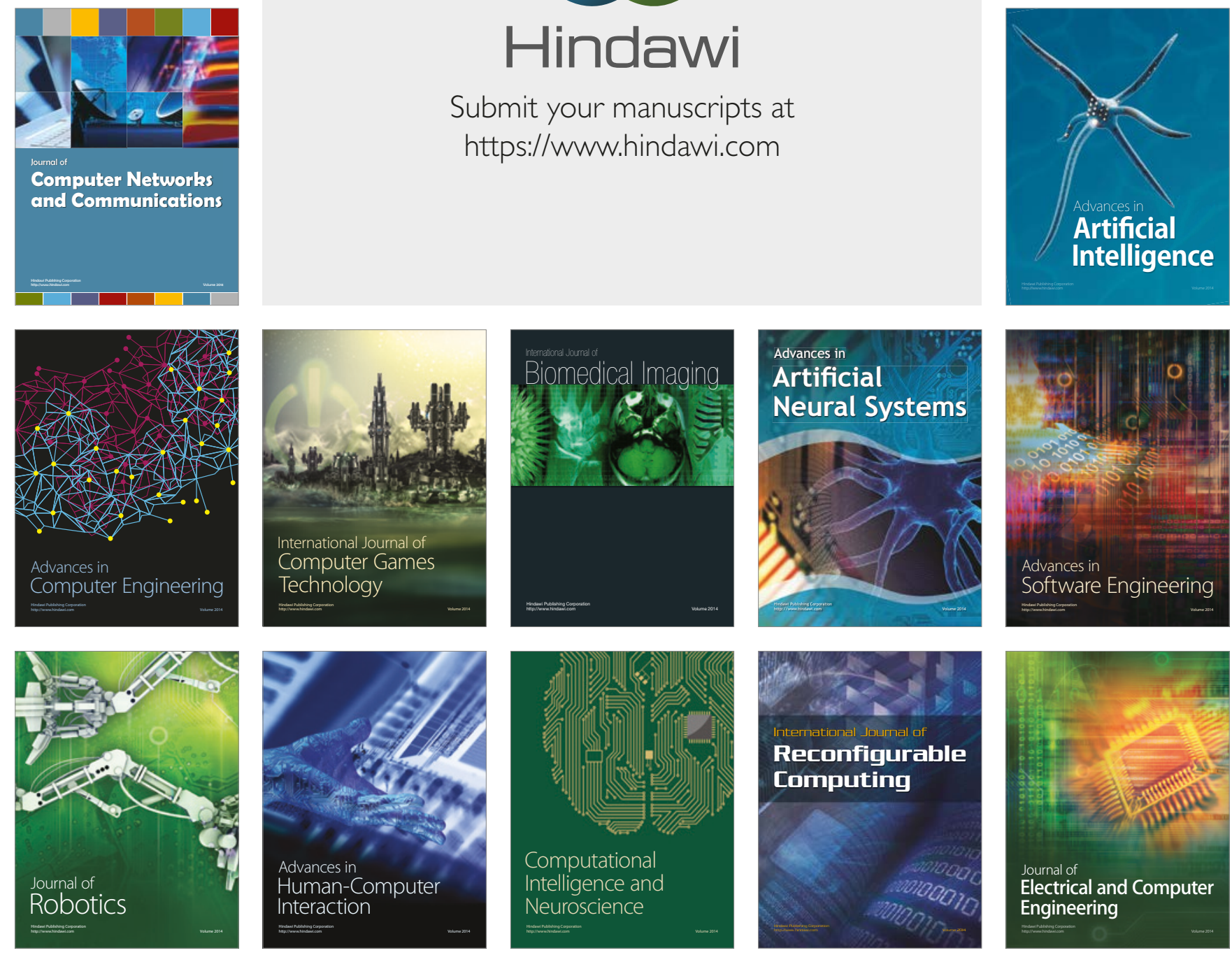